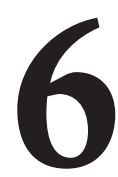

\title{
RECURSOS DIGITALES Y ENFOQUES DE ENSEÑANZA EN LA FORMACIÓN INICIAL DEL PROFESORADO DE HISTORIA ${ }^{1}$
}

\section{(DIGITAL RESOURCES AND TEACHING APPROACHES IN PRESERVICE TRAINING OF HISTORY TEACHERS)}

Cosme Jesús Gómez Carrasco

Universidad de Murcia

Jairo Rodríguez-Medina

Universidad de Valladolid

Álvaro Chaparro Sainz

Universidad de Almería

Santiago Alonso García

Universidad de Granada

DOI: 10.5944/educXX1.30483

Cómo referenciar este artículo/How to reference this article:

Gómez Carrasco, C.J., Rodríguez-Medina, J., Chaparro Sainz, Á. y Alonso García, S. (2022).

Recursos digitales y enfoques de enseñanza en la formación inicial del profesorado de Historia.

Educación XX1, 25(1), 143-170. https://doi.org/10.5944/educXX1.30483

Gómez Carrasco, C.J., Rodríguez-Medina, J., Chaparro Sainz, Á., \& Alonso García, S. (2022). Digital resources and teaching approaches in preservice training of History teachers. Educación XX1, 25(1), 143-170. https://doi.org/10.5944/educXX1.30483

\section{RESUMEN}

El presente trabajo se aborda teniendo en cuenta los estudios que demuestran la vinculación que realizan los docentes en formación con la función lúdica o motivacional concedida a las TIC y a los recursos digitales, así como a partir de las investigaciones que exponen la asociación existente entre el enfoque de enseñanza centrado en el docente (ITTF) con un aprendizaje superficial y el enfoque centrado en el alumnado (CCSF) con un aprendizaje profundo.

Sobre este enunciado, se plantea como objetivo general analizar los enfoques de enseñanza del futuro profesorado de historia en España y su relación con sus opiniones sobre el uso de recursos digitales en un aula. Para ello, se utilizó un diseño cuantitativo no experimental, con una escala 
Likert, en el que participaron 646 estudiantes del Máster de Formación del Profesorado de la especialidad de Geografía e Historia de 22 universidades nacionales diferentes, es decir, el 70\% de los centros de Educación Superior que ofertan dicha titulación en España.

Como conclusión general, el análisis estadístico realizado con los datos correspondientes a los enfoques del futuro profesorado de historia en España y su relación con sus opiniones sobre el uso de recursos digitales en un aula permite inferir que la validación y la fiabilidad de la herramienta aplicada es muy positiva; que existe una débil vinculación de los recursos digitales con los procesos educativos y; por último, que se destaca una relación significativa entre la aplicación de un enfoque de enseñanza concreto y una visión particular acerca del uso de los recursos tecnológicos.

\section{PALABRAS CLAVE}

Enseñanza de la historia, Recursos didácticos, Tecnología educativa, Formación inicial del profesorado, Máster de Educación Secundaria

\section{ABSTRACT}

The present research is approached taking into account the studies that demonstrate the connection that teachers in training make with the playful or motivational role granted to ICT and digital resources, as well as from the research shown by the association between a teacher-centred teaching approach (ITTF) with superficial learning and a student-centred approach (CCSF) with deep learning.

Thus, the general objective of this research is to analyze the teaching approaches of future history teachers in Spain and their relationship with their opinions on the use of digital resources in a classroom. For this purpose, a non-experimental quantitative design with a Likert scale was used, in which 646 students of the Master's Degree in Teacher Training in the specialty of Geography and History from 22 different national universities participated, that is, $70 \%$ of the universities offering this degree in Spain.

As a general conclusion, the statistical analysis carried out with the data corresponding to the approaches of future history teachers in Spain and their relationship with their opinions on the use of digital resources in a classroom allows us to infer that the validation and reliability of the applied tool is very positive; that there is a weak link between digital resources and educational processes and; finally, a significant relationship between the 
application of a particular teaching approach and a particular view about the use of technological resources is highlighted.

\section{KEY WORDS}

History Education, Teaching Resources, Educational Technology, Preservice Teacher Education, PGCE

\section{INTRODUCCIÓN}

La búsqueda de una mejora de la calidad educativa en todos los niveles se ha incrementado en los últimos años de manera exponencial. Este camino a la excelencia del sistema educativo ha traído consigo la necesidad de focalizar el prisma de análisis sobre el profesorado y sus enfoques de enseñanza como uno de sus principales campos de investigación. No en vano, los enfoques de enseñanza están indefectiblemente relacionados con la metodología de los docentes, así como con los principios epistemológicos que estos sostienen (Dejene et al., 2018; Miralles et al., 2019; Yunga-Godoy et al., 2016).

En este marco de investigación, instrumentos como el cuestionario ATI (Approaches to Teaching Inventory), desarrollado por Trigwell y Prosser (1996), se ubican en el centro del campo de estudio, dado que su uso otorga información sobre las perspectivas didácticas del profesorado (Yunga-Godoy et al., 2016). Los estudios realizados mediante la aplicación de este instrumento han permitido identificar preferentemente dos enfoques de enseñanza diferentes: uno centrado en la transmisión de la información por parte del docente (ITTF) y otro centrado en el cambio conceptual de los estudiantes (CCSF) (Dejene et al., 2018).

Pese a la identificación de estos dos grandes nodos, algunos autores señalan la existencia de categorías intermedias (Soler et al., 2018), por lo que en la práctica pueden observase docentes que prefieren los ítems que caracterizan o definen un enfoque, pero sin rechazar de plano los que configuran el otro factor (Yunga-Godoy et al., 2016). De esta manera, existen variables que pueden condicionar una acción docente centrada en el cambio conceptual de los estudiantes (CCSF) (e. g. la ajustada carga de trabajo); mientras que, por otro lado, hay variables que impulsan un enfoque centrado en la mera transmisión de la información por parte del profesor (ITTF) (e. g. la falta de compromiso del alumnado). 
Ante esta situación, consideramos determinante investigar sobre los enfoques de enseñanza que posee el actual profesorado en formación de Educación Secundaria en España, a sabiendas de que este se construye a partir de la experiencia vivida durante su formación (Martínez et al., 2009). De hecho, recientes indagaciones en el ámbito español apuntan a que la mayoría de los futuros docentes reconocían haber aprendido a través de estrategias y técnicas tradicionales donde primaba la información transmitida por el docente y/o el libro de texto, potenciando la memorización y el aprendizaje histórico como un saber acabado (Gómez, Miralles, et al., 2020; Gómez et al., 2018).

En este contexto, un importante número de investigaciones han mostrado la diversidad de realidades existentes en relación con cómo el futuro profesorado expresa su intención de implementar metodologías activas e innovadoras (De Groot-Reuvekamp et al., 2018; García-Martín y GarcíaSánchez, 2017; Gómez et al., 2019; Moreno-Guerrero et al., 2020; RodríguezMedina et al., 2020), si bien la tendencia real sigue mostrando la continuidad de las actuaciones más tradicionales, lo que justifica la necesidad de cambiar las concepciones para revertir dicha situación (König et al., 2017).

Esta ruptura del cambio de concepciones es innegociable dado que el enfoque de enseñanza adoptado por el profesorado influye en la manera de aprender los contenidos por parte del estudiante, al tiempo que el enfoque de aprendizaje adoptado por este determina igualmente su formación (Gargallo et al., 2015). De hecho, se ha demostrado la asociación existente entre el enfoque de enseñanza centrado en el docente (ITTF) con un aprendizaje superficial, al tiempo que un enfoque centrado en el alumnado (CCSF) se relaciona preferentemente con un aprendizaje profundo (Trigwell y Prosser, 1996). Una realidad que se encuentra relacionada con tres variables que deben ser tenidas en cuenta: los estudiantes y su ruta de aprendizaje, los docentes y su ruta de enseñanza y, por último, el contexto en el que se produce este proceso formativo (Guillermo et al., 2018).

Esta realidad cobra más relevancia en el contexto presente ante la translación que se está produciendo como consecuencia de la digitalización, ya que algunas investigaciones han llegado a considerar que la implementación de la tecnología en las aulas no ha sido real debido a la escasa formación del profesorado (Claro et al., 2018; Ramírez-García y González-Fernández, 2016). Una situación que ha quedado demostrada de forma empírica ante el escenario de enseñanza online que, de manera generalizada, se está implantando en los centros educativos como consecuencia de la pandemia mundial por la COVID-19.

Sin embargo, aun en el presente, los estudios demuestran la vinculación que realizan los docentes en formación con la función lúdica 
o motivacional concedida a las TIC y a los recursos digitales, otorgando una utilidad mayormente anecdótica (Hatlevik y Hatlevik, 2018; Miralles, Gómez, y Monteagudo, 2019). De hecho, las valoraciones que el profesorado realiza de esta herramienta no recogen sus posibilidades para el desarrollo en el alumnado de la capacidad de evaluar información digital, de resolver interrogantes históricos o de formar una ciudadanía reflexiva (Miralles et al., 2019). Es decir, permanecen sin ser abordadas las mejoras pedagógicas sobre la práctica docente que pudiera incorporar la implementación de la tecnología en los procesos de enseñanza-aprendizaje en la asignatura de Historia (Rivero y Mur, 2015).

Por este motivo, se plantea la necesidad de una investigación que se centre, no solo, en conocer los enfoques de enseñanza del profesorado en formación inicial sobre la materia de Historia con el fin de mejorar la capacitación docente en lo concerniente a la metodología, así como al enfoque epistemológico de dicha disciplina; sino también en analizar su opinión sobre el uso de los principales materiales y recursos digitales al alcance de un docente (Arancibia y Badia, 2015). Este tipo de estudios posibilita recabar información útil de cara a reforzar los programas de formación del profesorado en competencias digitales docentes de forma que se pueda llegar más allá del simple conocimiento de las herramientas TIC (Miralles et al., 2019).

\section{METODOLOGÍA}

\section{Objetivos}

El objetivo general (OG) de esta investigación consiste en analizar los enfoques de enseñanza del futuro profesorado de historia en España y su relación con sus opiniones sobre el uso de recursos digitales en un aula. De dicho OG se desglosan dos objetivos específicos (OE):

1. Detallar las opiniones del futuro profesorado de historia sobre el uso de recursos digitales en Educación Secundaria.

2. Analizar los perfiles de respuesta y sus diferencias con respecto a los enfoques de enseñanza que manifiestan en el cuestionario ATI (Approaches Teaching Inventory). 


\section{Diseño}

Se utilizó un diseño cuantitativo no experimental, con recopilación de información a través de un cuestionario con una escala Likert (1-5). Se eligió este tipo de diseño porque es capaz de responder a los problemas tanto en términos descriptivos como en relación con las variables, cuando la información se recopila sistemáticamente.

\section{Población y muestra}

La población del estudio es el alumnado de la especialidad de Geografía e Historia del Máster de Formación del Profesorado. Participaron 646 estudiantes de 22 universidades diferentes: Murcia, Alicante, Valencia, Jaume I, Barcelona, La Rioja, Zaragoza, Oviedo, País Vasco, Santiago de Compostela, Complutense de Madrid, Autónoma de Madrid, Valladolid, Extremadura, Castilla-La Mancha, Huelva, Sevilla, Córdoba, Málaga, Almería, Jaén y Granada. La muestra de esta investigación ha implicado al 70\% de la población. La muestra es incidental. Se contactó con los coordinadores de la especialidad y con el profesorado que impartía las materias específicas de didáctica de las ciencias sociales del Máster.

\section{Herramienta de recogida de datos}

Los datos se han recogido de una batería de instrumentos titulada "Enfoques de enseñanza en educación histórica". En la primera parte de esta batería se ubica el cuestionario ya validado ATI, a partir de su versión de 22 ítems (Trigwell et al., 2005). En la segunda parte de esta batería de instrumentos, se interroga a los participantes sobre sus opiniones acerca de las temáticas, recursos, competencias y métodos más adecuados para enseñar historia. En esta investigación utilizaremos el cuestionario ATI y el Bloque II.3. de ítems de la segunda parte de la batería de instrumentos (documento suplementario).

En cuanto al cuestionario ATI, hemos utilizado la traducción realizada por Monroy et al. (2015), donde siguieron este procedimiento de traducción: el inventario fue traducido al español de forma independiente por el investigador (un hablante nativo de español) y por dos hablantes nativos de inglés no asociados con la investigación. Se compararon las tres traducciones al español para producir una versión final; se introdujeron ligeros cambios para mantener el significado del original (Monroy et al., 2015). El procedimiento de traducción de la versión final de ATI propuesta en este estudio (S-ATI-20) fue similar al procedimiento descrito anteriormente. 
Se realizaron pequeños cambios en la redacción de algunos ítems para su comprensión en español y la especificidad del contexto teniendo en cuenta la cultura educativa española y los cambios recientes introducidos en el Espacio Europeo de Educación Superior (EEES). Similar a Stes et al. (2010), se reformularon elementos en los que se sospechaba que existía ambigüedad en cuanto a si transmitían una noción de la ITTF o de la CCSF. La formulación de los ítems fue también adaptada a la materia de historia.

La validación del contenido del Bloque II.3 de ítems de la batería de instrumentos se realizó a través de siete expertos. Fueron cuatro expertos del área de Didáctica de las Ciencias Sociales, un experto del área de Didáctica y Organización Escolar, un experto del área de Métodos de Investigación y Diagnóstico en Educación, y otro experto del área de Psicología de la Educación. Esta validación se realizó a través de un cuestionario en el que los expertos debían valorar la suficiencia, claridad y pertinencia de los ítems con una escala de estimación del 1-4. En el caso de los ítems del Bloque II.3, los resultados fueron satisfactorios tanto en suficiencia $(\mathrm{M}=3.57$; $\mathrm{SD}$ $=.12) \mathrm{y}$ en claridad $(\mathrm{M}=3.85 ; \mathrm{SD}=.13)$ como en pertinencia $(\mathrm{M}=3.85$; $\mathrm{SD}=.23)$. Los coeficientes ponderados de concordancia de Bangdiwala $\left(B^{\mathrm{W}}{ }_{\mathrm{N}}\right)$ (Bangdiwala, 1987) obtenidos fueron excelentes en las tres variables analizadas (suficiencia $B^{\mathrm{W}}{ }_{\mathrm{N}}=.929$; claridad $B_{\mathrm{N}}^{\mathrm{W}}=.901$; pertinencia $B_{\mathrm{N}}^{\mathrm{W}}=.931$ ) así como a nivel global $\left(B_{\mathrm{N}}^{\mathrm{W}}=.920\right)$.

Para la realización de un análisis de fiabilidad del cuestionario ATI empleamos el método de consistencia interna basado en el alfa de Cronbach. Este análisis permite estimar la fiabilidad de un instrumento de medida compuesto por un conjunto de ítems, de tipo escala Likert 5, que esperamos midan la misma dimensión teórica (el mismo constructo). De esta manera, los ítems resultan sumables en una puntuación única que mide un rasgo que es importante en la construcción teórica del instrumento. La fiabilidad de la escala debe obtenerse siempre con los datos de cada muestra para garantizar la medida fiable del constructo en la muestra concreta de investigación. Como criterio general el coeficiente debe ser superior a .70 (George y Mallery, 2011). En el caso del cuestionario ATI a nivel global se obtuvo un coeficiente de alfa ordinal de .78, por lo que podemos considerar el coeficiente como aceptable. En este mismo sentido, otros autores proponen el coeficiente omega, también conocido como Rho de Jöreskog (VenturaLeón y Caycho-Rodríguez, 2017), y como criterio general se considera que hay que obtener un coeficiente mayor de .70. En nuestro caso, el coeficiente de omega de McDonald para el global de la escala es de .79, por lo que obtenemos un valor aceptable. Por su parte, en el caso del Bloque II.3 de ítems obtenemos un índice de alfa de Cronbach de .82 y omega de McDonald .83 , considerados ambos igualmente adecuados. 
Para ahondar en la validación del constructo del ATI se procedió a hacer un SEM (Structural Equation Modeling), para confirmar la existencia de una serie de constructos en el cuestionario. En un modelo de ecuaciones estructurales, en el fondo lo que estamos haciendo es una regresión logística, donde la variable dependiente sería el constructo (cada uno de los bloques del cuestionario), y los ítems serían las variables independientes. Al calcular el modelo se estiman los valores para esos coeficientes.

Se compara la matriz de covarianza derivada de las variables observadas y la matriz de covarianzas reproducida por el modelo. Así, se contrasta la hipótesis de que la diferencia entre la matriz procedente de los datos recogidos con el cuestionario y la matriz teórica definida en el modelo conceptual no es estadísticamente significativa. Al tratarse de una escala Likert, no se cumple el supuesto de normalidad, por lo que se opta por hacer una estimación robusta del estadístico $\chi 2$ mediante el estimador DWLS (Diagonal Weighted Least Squares), es decir, un estimador ponderado de mínimos cuadrados (Beaujean, 2014).

Para establecer los índices de ajustes del modelo se utilizan los valores de TLI (Tucker-Lewis Index), también conocido como NNFI (Non-Normed Fit Index) y de CFI (Comparative Fit Index), que toman valores entre 0 y 1 , indicando un buen ajuste aquellos valores cercanos a 1 (Kenny, 2012). Por otra parte, el valor de RMSEA (Root Mean Square Error of Approximation) mide la diferencia absoluta entre la estructura de relaciones, entre el modelo teórico propuesto y los datos observados, teniendo en cuenta el número de estimadores y el tamaño muestral (Steiger, 1990); tomando valores entre 0 y 1 , indicando un buen ajuste aquellos valores cercanos a 0 .

Se realiza el test de Bartlett para averiguar que la matriz no sea similar a una matriz identidad. Obtenemos un valor $\mathrm{p}<0.05$, por lo que nos indica que la matriz no es similar a la matriz identidad. Además, obtenemos un valor de la KMO de .84, lo que nos muestra que la matriz es factorizable.

En primer lugar, se realizó el análisis con el constructo teórico inicial del ATI. Tras separar ambas escalas revisamos la fiabilidad, obteniendo unos resultados adecuados. Para la escala CCS un alfa ordinal $=.87 \mathrm{y} \omega \mathrm{t}$ $=.87$. En el caso de la escala ITT obtenemos un alfa ordinal $=.72 ; \mathrm{y} \omega \mathrm{t}=.74$. La relación entre ambas escalas es negativa baja (-.15) pero significativa. El índice de ajuste de este modelo es aceptable $\left(\chi^{2}(169)=674.68, \mathrm{p}<.001\right.$, $\mathrm{CFI}=.936$, TLI=.928, RMSEA=.07). No obstante, aunque el modelo obtiene un ajuste aceptable, se decide eliminar el ítem 12 dado que aporta muy poco al factor ITT. Como consecuencia, los índices mejoran de forma sensible $\left(\chi^{2}(151)=478.93, \mathrm{p}<.001, \mathrm{CFI}=.957, \mathrm{TLI}=.951, \mathrm{RMSEA}=.059\right)$. En el caso de la fiabilidad, para la escala CCS seguimos obteniendo un alfa ordinal $=.87 \mathrm{y}$ 
$\omega \mathrm{t}=.87$. En el caso de la escala ITT mejoramos hacia un alfa ordinal $=.74 ; \mathrm{y}$ $\omega \mathrm{t}=.75$. En la Figura 1 se muestra la representación gráfica del modelo. La relación entre ambas escalas es negativa, significativa, pero con una carga factorial baja (-.17). No existe un continuo único bipolar entre el enfoque basado en la materia y enfoque basado en el alumnado. La preferencia por uno u otro funciona como un binomio de dos factores distintos y poco dependientes, de forma que pueden observarse personas que prefieren los ítems de un factor, pero no por eso rechazan los ítems del otro factor. Estos resultados son similares a los obtenidos en otros trabajos en los que se han analizado las propiedades métricas y la estructura factorial de la escala ATI (Monroy et al., 2015; Prosser y Trigwell, 2006).

\section{Figura 1}

Representación del modelo estructural

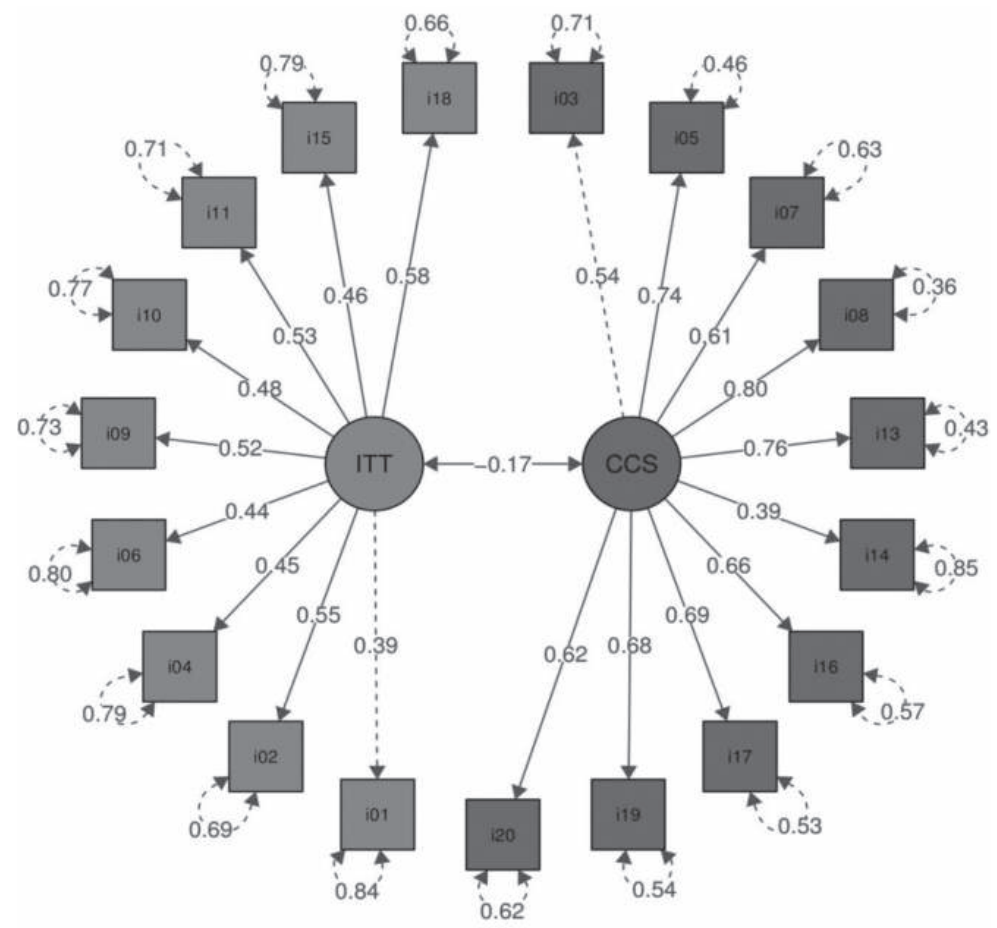

Nota. Elaboración propia

Los ítems 5, 8, 13 y 19 son los que más aportan al modelo CCS: "Se tiene que reservar parte del tiempo para que los estudiantes puedan discutir entre ellos conceptos e ideas claves de historia"; "En las sesiones de clase hay que planificar e impulsar debates y discusiones"; "Hay que intentar que los estudiantes discutan sus cambios de opinión y de comprensión 
de la historia"; "La enseñanza de esta asignatura tendría que ayudar a los estudiantes a cuestionar su propia comprensión de la historia". Como se puede comprobar, los ítems que más aportan al modelo están relacionados con la creación de debates, la discusión y el cuestionamiento de ideas previas.

En el caso del modelo ITT, los ítems 2, 9, 11 y 18 son los que más aportan al factor: "Para evaluar a los estudiantes se les debe pedir que describan todos los contenidos de historia que corresponden a los objetivos específicos y con lo que han de responder en su evaluación"; "Las clases de historia deben servir para que los estudiantes superen la evaluación"; "Hay que proporcionar a los estudiantes la información que necesitan para superar la asignatura de Historia"; "La forma de enseñar historia se centra en transmitir a los estudiantes mis conocimientos". Es decir, son los ítems relacionados con la transmisión de los conocimientos necesarios para que el alumnado pueda superar la asignatura de historia.

En lo que se refiere al Bloque II.3, para garantizar la calidad de la medida, se realizaron análisis factoriales exploratorios y confirmatorios. En primer lugar, se dividió la muestra original en dos submuestras aleatorias de $n=323$ participantes cada una, y se llevó a cabo un análisis factorial exploratorio (AFE) sobre la matriz de correlaciones policóricas entre los ítems (Hair et al., 2010). Tras comprobar la adecuación de los datos para el análisis factorial mediante las pruebas de Kaiser Meyer Olkin (KMO) y esfericidad de Barlett (KMO =.852; prueba de esfericidad de Bartlett, $\chi^{2}(120)$ $=4316.1 ; \mathrm{p}<0.001)$ se analizó la dimensionalidad de la escala mediante análisis paralelo (Timmerman y Lorenzo-Seva, 2011). A continuación, sobre la matriz de correlaciones policóricas obtenidas con la segunda submuestra aleatoria $(n=323)$ se estimaron los modelos confirmatorios. Para evaluar la adecuación del ajuste del modelo se emplearon los índices señalados anteriormente (TLI, CFI, RMSEA).

El análisis paralelo optimizado sobre 1000 réplicas aleatorias alcanzó una solución óptima de tres factores cuando se consideró el percentil 95 de la proporción de varianza aleatoria y de cuatro factores al considerar su media. La solución de cuatro factores seleccionada explica el 63.7\% de la varianza común. El primer factor, con un valor propio de 5.8, explica el $36.3 \%$ de la varianza común, se ha denominado recursos patrimoniales y está constituido por 5 ítems $(56 ; 61 ; 62 ; 64 ; 65)$. El segundo factor, con un valor propio de 1.65 , explica el $10.3 \%$ de la varianza común, se ha denominado fuentes y está constituido por 3 ítems $(52 ; 53 ; 54)$. El tercer factor, con un valor propio de 1.56, explica el $9.75 \%$ de la varianza común, se ha denominado recursos tradicionales y está constituido por 2 ítems (50; 55). Por último, el cuarto factor, con un valor propio de 1.17 , explica el $7.3 \%$ de la varianza común, se ha denominado recursos digitales/mass media y está constituido por 6 ítems 
(51; 57; 58; 59; 60; 63). Finalmente, se comparó la bondad del ajuste de cuatro modelos alternativos de uno, dos, tres y cuatro factores. El resultado fue muy favorable al de cuatro factores correlacionados $\left(\chi^{2}(99)=282.83, \mathrm{p}\right.$ $<.001, \mathrm{CFI}=.956, \mathrm{TLI}=.947$, RMSEA=.054) (ver Tabla S1 y Figura S1). En lo que se refiere a la fiabilidad de cada una de las subescalas los resultados obtenidos fueron también adecuados (recursos patrimoniales $\alpha=.87, \omega=.84$; fuentes $\alpha=.75, \omega=.68$; recursos tradicionales $\alpha=.68, \omega=.67$; recursos digitales/ mass media $\alpha=.79, \omega=.74)$.

\section{Procedimiento y análisis de datos}

La investigación contó con la aprobación del comité de ética de la Universidad de Murcia. Para recoger información (material suplementario), se envió una carta en la que se explicaban los objetivos del proyecto ${ }^{1}$ a los coordinadores del Máster, al tiempo que se les facilitó el enlace web a través del cual el alumnado podría participar. En la primera página de ese enlace se incluía un documento de consentimiento informado para los participantes antes de comenzar la encuesta a la cual respondieron desde noviembre de 2019 a febrero de 2020.

El análisis de datos se ha realizado en tres fases: a) análisis descriptivo del Bloque II.3 de la batería de instrumentos para conocer las respuestas del profesorado sobre los recursos y materiales más adecuados para la enseñanza de la historia; b) análisis de Clúster Aglomerativo Jerárquico para establecer los perfiles de respuesta del profesorado en formación sobre los recursos y materiales más adecuados para la enseñanza de la historia; y c) Análisis inferencial (ANOVA de un factor) entre los perfiles de respuesta y las puntuaciones medias del factor CCSF e ITTF. Todos estos análisis se han realizado con la librería de R lavaan (Rosseel, 2012) y el programa XLSTAT en su versión 2020.3.1.

\section{RESULTADOS}

\section{Análisis descriptivo}

En la Tabla 1 y la Figura 2 pueden observarse los estadísticos descriptivos del Bloque II.3 de la batería de instrumentos, en el que los docentes en formación evaluaban qué recursos consideraban más apropiados para la enseñanza de la Historia. De este modo, si tomamos como pauta la valoración media otorgada por los participantes en la encuesta a cada uno de los ítems, los recursos que el futuro profesorado de historia considera más apropiados (por encima de 4 de media) son: patrimonio histórico y cultural próximo 
(Ítem 65), con un 4.61; museos y otros lugares de interpretación patrimonial (Ítem 56) con un 4.49, producciones artísticas (Ítem 64) con un 4.45, fuentes documentales primarias (ítem 52) con 4.27, cine y documentales de contenido histórico (ítem 57) con 4.19, recreaciones virtuales en museos (ítem 62) con 4.12 y, finalmente, fuentes orales (ítem 53) con una valoración media de 4.11.

En el lado opuesto, los recursos que el futuro profesorado de historia considera menos adecuados (debajo de 3.5 de valoración) son el libro de texto (Ítem 50), con un 2.61 y los videojuegos (Ítem 60), con un 3.24. Por último, recursos digitales y de mass-media, como páginas web de contenido histórico (3.61) o aplicaciones para teléfono móvil y tabletas con contenido histórico (3.65) tienen una valoración moderada, igual que los apuntes del profesor (3.64). Los recursos relacionados con el patrimonio (museos, patrimonio próximo, producciones artísticas, recreaciones virtuales en museos...) y los de carácter procedimental (fuentes documentales y orales), tienen una valoración más alta. Los recursos digitales tienen una valoración más baja, excepto el uso del cine y las recreaciones virtuales en museos.

Tabla 1

Estadísticos Descriptivos sobre la Opinión del Uso de Recursos en la Clase de Historia

\begin{tabular}{cccccccccccc}
\hline Ítem & $\mathbf{N}$ & Min & Máx & Media & Mediana & $\% 1$ & $\% \mathbf{2}$ & $\% \mathbf{3}$ & $\% \mathbf{4}$ & $\% \mathbf{5}$ & $\mathbf{S D}$ \\
\hline 50 & 644 & 1 & 5 & 2.93 & 3.00 & 9.01 & 23.40 & 38.20 & 23.90 & 5.43 & 1.02 \\
\hline 51 & 645 & 1 & 5 & 3.61 & 4.00 & 1.86 & 7.44 & 32.90 & 43.30 & 14.60 & .89 \\
\hline 52 & 645 & 1 & 5 & 4.27 & 4.00 & .16 & 4.19 & 11.20 & 37.40 & 47.10 & .83 \\
\hline 53 & 645 & 1 & 5 & 4.11 & 4.00 & .62 & 4.19 & 18.10 & 37.50 & 39.50 & .89 \\
\hline 54 & 643 & 1 & 5 & 3.82 & 4.00 & 1.09 & 4.82 & 27.10 & 45.10 & 21.90 & .87 \\
\hline 55 & 642 & 1 & 5 & 3.64 & 4.00 & 1.25 & 7.94 & 34.10 & 39.10 & 17.60 & .90 \\
\hline 56 & 643 & 2 & 5 & 4.49 & 5.00 & .00 & .78 & 6.22 & 35.80 & 57.20 & .65 \\
\hline 57 & 643 & 1 & 5 & 4.19 & 4.00 & .16 & 2.02 & 14.00 & 46.70 & 37.20 & .76 \\
\hline 58 & 644 & 1 & 5 & 3.74 & 4.00 & 2.64 & 7.30 & 27.00 & 39.40 & 23.60 & .98 \\
\hline 59 & 644 & 1 & 5 & 3.87 & 4.00 & 1.71 & 5.28 & 24.40 & 41.90 & 26.70 & .93 \\
\hline 60 & 645 & 1 & 5 & 3.24 & 3.00 & 7.13 & 16.70 & 34.90 & 27.30 & 14.00 & 1.11 \\
\hline 61 & 644 & 1 & 5 & 3.86 & 4.00 & 1.40 & 5.28 & 27.20 & 38.70 & 27.50 & .93 \\
\hline 62 & 641 & 1 & 5 & 4.12 & 4.00 & .62 & 3.28 & 16.40 & 42.60 & 37.10 & .84 \\
\hline 63 & 644 & 1 & 5 & 3.65 & 4.00 & 4.19 & 7.14 & 27.60 & 41.30 & 19.70 & 1.01 \\
\hline 64 & 644 & 1 & 5 & 4.45 & 5.00 & .31 & .47 & 8.70 & 34.50 & 56.10 & .70 \\
\hline & & & & & & & & & & &
\end{tabular}




\begin{tabular}{cccccccccccc}
\hline Ítem & $\mathbf{N}$ & Min & Máx & Media & Mediana & $\% 1$ & $\% \mathbf{2}$ & $\% 3$ & $\% \mathbf{4}$ & $\% 5$ & $\mathbf{S D}$ \\
\hline 65 & 645 & 2 & 5 & 4.61 & 5.00 & .00 & .46 & 5.58 & 26.80 & 67.10 & .62 \\
\hline Total & 643.35 & 1 & 5 & 3.91 & 4.06 & 2.01 & 6.29 & 22.10 & 37.58 & 32.02 & .87 \\
\hline
\end{tabular}

Nota. Elaboración propia

\section{Figura 2}

Frecuencias sobre la opinión del uso de recursos en la clase de historia

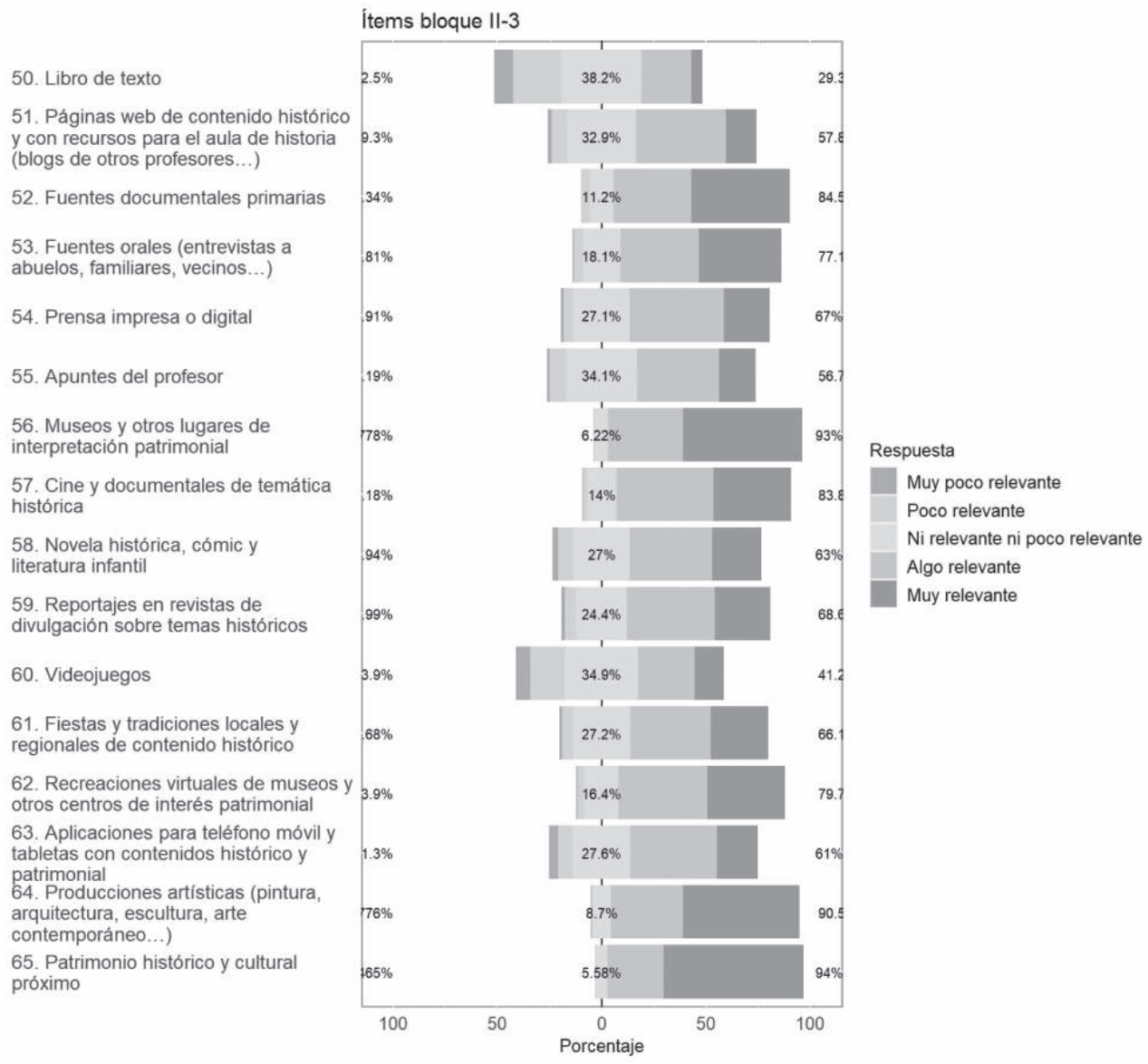

Nota. Elaboración propia 


\section{Análisis de perfiles}

Para delimitar los perfiles de respuesta se ha optado por un análisis de Clúster Aglomerativo Jerárquico (CAJ) con el método de Ward, obteniéndose como resultado tres clases. Los datos nos indican que la clase más numerosa es la clase 1 (246 participantes), seguida de la clase 3 (220) y la clase 2 (180). La clase 1 es la que tiene más varianza (12.4) y la que tiene mayor distancia con el centroide. Como se puede apreciar en el dendrograma (Figura 3), esta clase 1 está más alejada de las otras dos clases, que están más relacionadas.

\section{Figura 3}

Dendograma

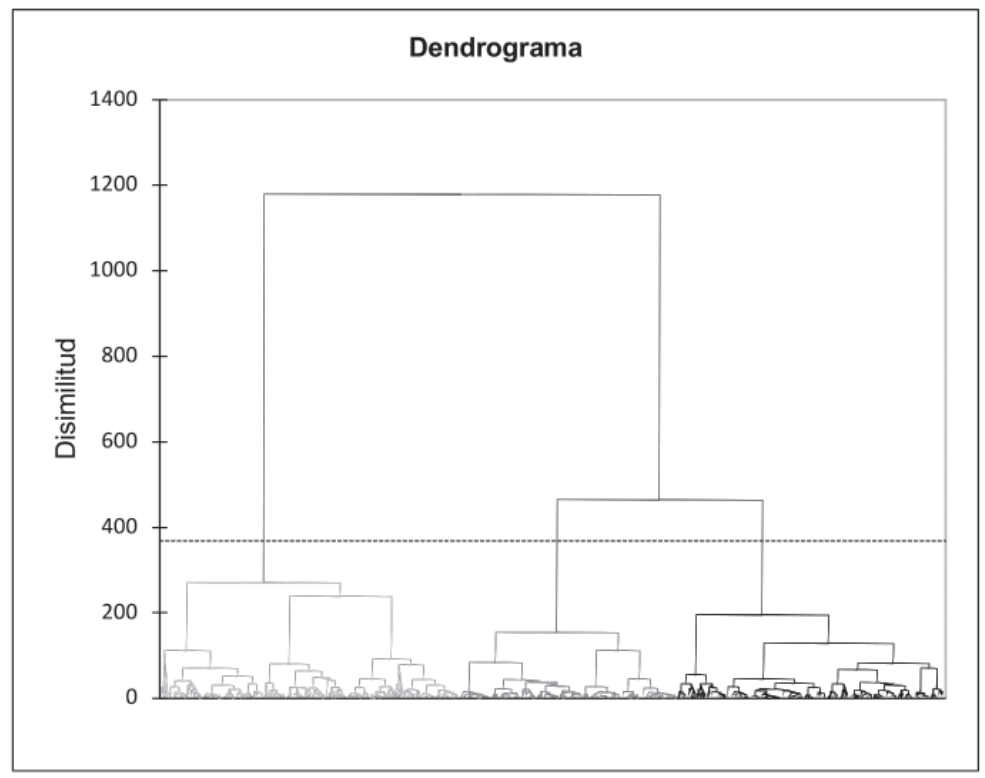

Nota. Elaboración propia

Se han agrupado las puntuaciones de los ítems en los cuatro factores resultados del SEM del Bloque II.3 (fuentes, patrimonio, tradicional y digital). Para comprobar las diferencias entre las clases se ha realizado un análisis multivariante de la varianza (MANOVA). Se observaron diferencias estadísticamente significativas entre las tres clases según la puntuación obtenida en los cuatro factores (Lambda de Wilks $=0.368, \mathrm{~F}(8.1280)=$ 103.86, $p<.01)$. Para complementar el MANOVA se han realizado cuatro análisis univariados de la varianza (ANOVA) entre las tres clases y las puntuaciones medias de los cuatro factores, así como un análisis post hoc a través de la prueba de Tukey. Los resultados se han resumido en la Tabla 2, 
indicando la significatividad y los grupos (a, b y c) en los que la prueba de Tukey colocó a cada una de las clases. Hay diferencias significativas entre las tres clases y las puntuaciones en el factor fuentes $(\mathrm{F}(2.643)=165, p$ $<.01)$, en el factor recursos patrimoniales $(\mathrm{F}(2.643)=186, p<.01)$, en el factor tradicional $(\mathrm{F}(2.643)=196, p<.01)$, y en el factor recursos digitales $(\mathrm{F}(2.643)$ $=273, p<.01)$.

\section{Tabla 2}

Resumen de los resultados de los cuatro ANOVA realizados entre los perfiles de las clases y las puntuaciones agrupadas de los factores del Bloque II. 3

\begin{tabular}{ccccc}
\hline Clase & Fuentes & Patrimonio & Tradicional & Digital \\
\hline 2 & $4.452 \mathrm{a}$ & $4.787 \mathrm{a}$ & $3.528 \mathrm{a}$ & $4.369 \mathrm{a}$ \\
\hline 3 & $4.309 \mathrm{~b}$ & $4.324 \mathrm{~b}$ & $3.032 \mathrm{c}$ & $3.654 \mathrm{~b}$ \\
\hline 1 & $3.543 \mathrm{c}$ & $3.896 \mathrm{c}$ & $3.295 \mathrm{~b}$ & $3.267 \mathrm{c}$ \\
\hline Pr $>\mathrm{F}($ Modelo $)$ & $<.001$ & $<.001$ & $<.001$ & $<.001$ \\
\hline Significativo & Sí & Sí & Sí & Sí \\
\hline
\end{tabular}

En el perfil de las clases agrupadas por factores (Figura 4) podemos comprobar cómo la clase 1 es la que puntúa significativamente más bajo los recursos digitales. Igualmente se puede apreciar cómo esta clase 1, a pesar de valorar generalmente más bajo a todos los recursos, la puntuación al factor tradicional es superior a la clase 3 y muy próximo a la clase 1 . Nos encontramos, pues, ante una clase de profesorado que no rechaza esos recursos tradicionales, pero sí puntúa significativamente más bajo el resto de los recursos. La clase 2 puntúa más alto todos los recursos, si bien destaca que su valoración del factor patrimonio y digital es muy superior al resto. La clase 2 apuesta más claramente por los recursos de innovación basados en la motivación del alumnado (recursos digitales y patrimonio próximo). La clase 3 presenta un perfil diferente, puesto que valora muy alto el factor fuentes (casi tan alto como la clase 2); sin embargo, puntúa significativamente más bajo que la clase 2 los recursos digitales. Así, mientras que en la clase 2 las diferencias entre el factor fuentes y digital es de -.1, en la clase 3 es de -.7. Además, esta clase puntúa muy bajo el factor tradicional, por lo que se infiere que apuesta preferiblemente por una innovación basada en recursos procedimentales (el uso de fuentes históricas en el aula), rechaza los modelos tradicionales de enseñanza y puntúa de forma moderada los recursos de innovación ligados a la motivación, como señalan otros artículos (Miralles et al., 2019). 


\section{COMPARACIÓN ENTRE PERFILES Y LAS PUNTUACIONES FACTORIALES}

Para ver las diferencias existentes entre los diferentes perfiles en función de los enfoques de enseñanza que manifiestan en el cuestionario ATI, se ha realizado un análisis inferencial a través de ANOVA de un factor con las puntuaciones factoriales del factor CCSF y del factor ITTF. De este modo, con respecto al factor CCSF, en el análisis inferencial a través de ANOVA encontramos diferencias significativas entre las clases $(F(2,643)=$ $24.08, p<.001$ ) (Figura 5).

\section{Figura 4}

Perfil de las clases agrupadas por factores

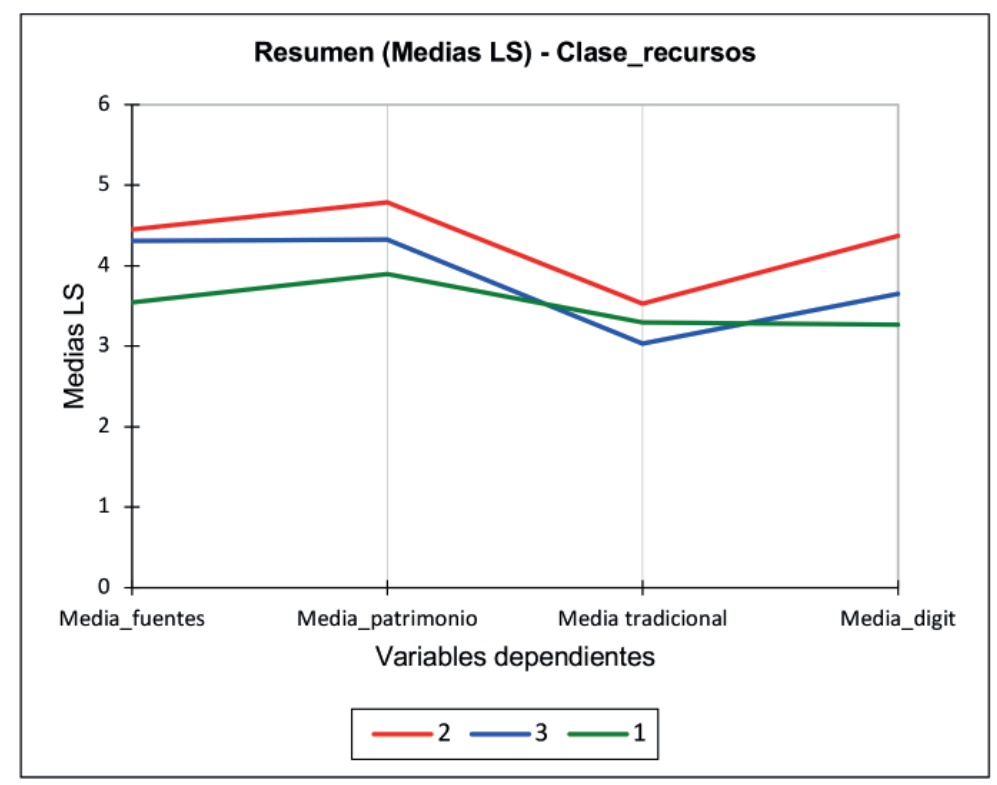

Nota. Elaboración propia 


\section{Figura 5}

Análisis inferencial a través de ANOVA

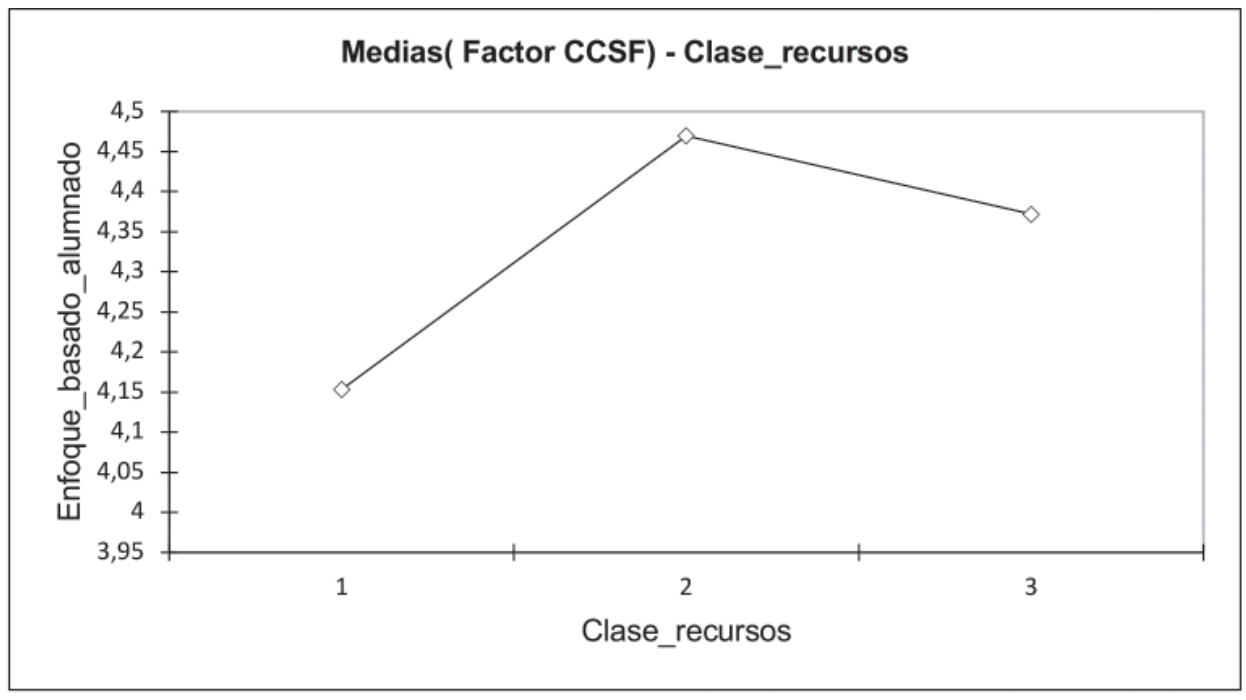

Nota. Elaboración propia

Se ha realizado la prueba de contraste Tukey (Tabla 3) para comprobar las diferencias entre clases. Las principales diferencias se dan entre la clase 1 con la clase 2 y 3 . No hay diferencias significativas entre las clases 2 y 3 en sus puntuaciones medias al factor CCSF. Así, es la clase 1 la que puntúa, de forma significativa, más bajo el factor CCSF. Esta clase también tenía una valoración más baja de los recursos digitales y de los recursos procedimentales, como las fuentes documentales y orales.

\section{Tabla 3}

Clase_recursos / Tukey (HSD) / Análisis de las diferencias entre las categorías con un intervalo de confianza de 95\% (Enfoque_basado_alumnado)

\section{Contraste Diferencia $\begin{gathered}\text { Diferencia } \\ \text { estandarizada }\end{gathered} \begin{gathered}\text { Valor } \\ \text { crítico }\end{gathered}$ Pr $>$ Dif Significativo}

\begin{tabular}{cccccc}
\hline 2 vs 1 & .316 & 6.615 & 2.349 & $<.0001$ & Sí \\
\hline 2 vs 3 & .098 & 1.993 & 2.349 & .115 & No \\
\hline 3 vs 1 & .219 & 4.833 & 2.349 & $<.0001$ & Sí \\
\hline
\end{tabular}

Nota. Elaboración propia 
Con respecto al enfoque de enseñanza ITTF basado en el profesor, en el análisis inferencial a través de ANOVA de dicho factor, encontramos diferencias significativas entre las clases $(\mathrm{F}(2.643)=6.34, p<.05)$ (Figura 6).

Se ha realizado la prueba de contraste Tukey (Tabla 4) para comprobar las diferencias entre clases. Las principales diferencias se dan entre la clase 3 con la clase 1 y 2 . No hay diferencias significativas entre las clases 1 y 2 en sus puntuaciones medias al factor ITTF. Así, es la clase 3 la que puntúa, de forma significativa, más bajo el factor ITTF. A pesar de ello, no hay grandes diferencias entre las clases. La clase 3, que puntúa muy alto (igual que la clase 2) los recursos procedimentales y de una forma intermedia la mayoría de los recursos digitales tiene un mayor rechazo el enfoque basado en la materia (2.94). Las mayores diferencias en el enfoque basado en la materia están entre la clase 1 (que no puntúa tan bajo los recursos tradicionales como libros de texto y apuntes, pero sí algunos de los recursos digitales) y la clase 3 (3.11 frente a 2.94). La clase 2, que es la que puntúa más alto el enfoque basado en el estudiante, tiene una posición más cercana a la clase 1 en el enfoque basado en la materia (3.1).

\section{Figura 6}

Análisis inferencial a través de ANOVA

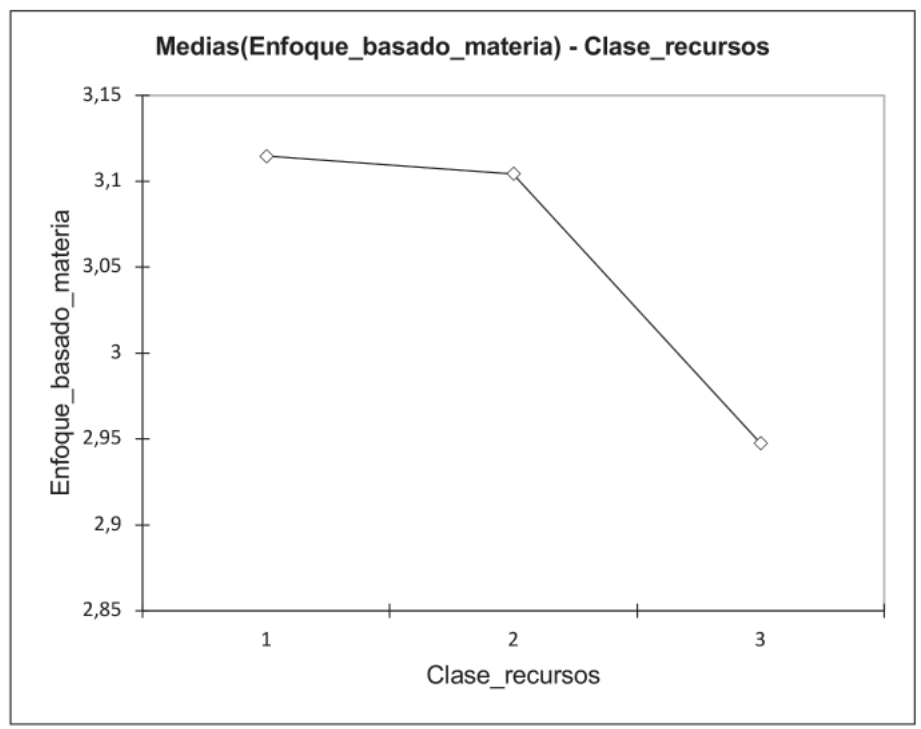

Nota. Elaboración propia 


\section{Tabla 4}

Clase_recursos / Tukey (HSD) / Análisis de las diferencias entre las categorías con un intervalo de confianza de 95\% (Enfoque_basado_materia)

\begin{tabular}{cccccc}
\hline Contraste & Diferencia & $\begin{array}{c}\text { Diferencia } \\
\text { estandarizada }\end{array}$ & $\begin{array}{c}\text { Valor } \\
\text { crítico }\end{array}$ & Pr $>$ Dif & Significativo \\
\hline 1 vs 3 & .167 & 3.267 & 2.349 & .003 & Sí \\
\hline 1 vs 2 & .010 & .192 & 2.349 & .980 & No \\
\hline 2 vs 3 & .157 & 2.829 & 2.349 & .013 & Sí \\
\hline
\end{tabular}

Nota. Elaboración propia

\section{DISCUSIÓN Y CONCLUSIONES}

Optar por la calidad requiere una continua búsqueda de mejora del ecosistema educativo en todos los niveles, hecho que se ha potenciado en los últimos años de manera exponencial en la educación superior tras el proceso de Bolonia (Zurita et al., 2017). En este punto, los principios epistemológicos y sus enfoques de enseñanza se convierten en uno de los aspectos primarios de análisis y mejora (King et al., 2019; Thompson, 2019). Así pues, a continuación, se presentan las correspondientes conclusiones específicas (CE1 y CE2), sobre la base del objetivo general (OG) y los objetivos específicos (OE) planteados al inicio de la investigación.

Así, respecto a las CE1, cabe señalar que las opiniones que manifestó el profesorado en formación inicial sobre el uso de recursos para la enseñanza de la historia, mostraban una valoración media o media-baja respecto a aquellos ítems más relacionados con elementos tecnológicos o multimedia. Una conclusión de la que se infiere que existe una débil vinculación de los recursos digitales con estrategias de enseñanza, puesto que estos se reservan prácticamente para cometidos motivacionales o de carácter lúdico, como han podido indagar en otros estudios (Hatlevik y Hatlevik, 2018; Miralles, Gómez y Monteagudo, 2019). De hecho, en la investigación se observa cómo el futuro profesorado de historia de España no solo consideran estos recursos como algo anecdótico, sino que - dentro de sus valoraciones-son los elementos que reciben las puntuaciones más bajas. No en vano, tres de los recursos que poseen un marcado carácter tecnológico se encuentran por debajo de la media del conjunto de ítems (3.91): páginas web de contenido histórico (3.61), videojuegos (3.24) y aplicaciones para teléfono móvil o tabletas con contenido histórico o patrimonial (3.65). Incluso el enunciado relativo a prensa impresa o digital, que también posee un componente tecnológico relevante dada la accesibilidad actual a los repositorios digitales, ha sido valorado por debajo de la mencionada media (3.82). De hecho, alguno de 
los ítems indicados se encuentra incluso peor valorado que los apuntes del profesor (3.64), un recurso estrechamente afín con un enfoque de enseñanza transmisivo y centrado en el docente. En este contexto, cabe señalar cómo únicamente el ítem 57 (cine y documentales de temática histórica), con una puntuación media de 4.19 y el ítem 62 (recreaciones virtuales de museos y otros centros de interés patrimonial), con una valoración de 4.12, fueron favorablemente considerados por el alumnado como recursos docentes válidos para la enseñanza de la historia dentro de un marco tecnológico.

Por lo que se refiere a las CE2, al analizar los perfiles de respuesta y sus diferencias con respecto a los enfoques de enseñanza que se manifiestan a través del cuestionario ATI, observamos cómo el perfil de clase 2 puntúa más alto todos los recursos, especialmente aquellos factores vinculados al patrimonio y a los recursos digitales. Por tanto, se trata de una clase que apuesta más claramente por los recursos de innovación basados en la motivación del alumnado. De hecho, existen diferencias estadísticamente significativas entre la clase 1, que es la que puntúa más bajo el factor CCSF centrado en el alumnado respecto de las clases 2 y 3 . En definitiva, la clase 2, que es la que puntúa más alto el enfoque basado en el estudiante (CCS), tiene una posición más cercana a la clase 1 en el enfoque basado en la materia (ITT). Por su parte, esta clase 1 es la que puntúa más bajo los recursos digitales y procedimentales, como el uso de fuentes, al tiempo que otorga una puntuación al factor "tradicional" superior a la clase 3 y próximo a la clase 2 . Se destaca, en conclusión, el hecho de que hay una relación significativa y directa entre la aplicación de un enfoque de enseñanza basado en el estudiante y una visión más positiva del uso de recursos tecnológicos (Miralles et al., 2019).

La introducción de nuevos recursos didácticos en las aulas de Educación Secundaria podría consolidar nuevas metodologías de enseñanza. Dar cobertura a nuevas estrategias, apoyándose en nuevos instrumentos, permite mejorar los procesos de aprendizaje, consiguiendo mejores resultados académicos (Gómez, Monteagudo, et al., 2020). Los resultados nos invitan a pensar en dos líneas. Por un lado, el futuro profesorado de historia reconoce la valía de los recursos más vinculados a sus disciplinas científicas de origen, dado que estamos ante futuros docentes que, preferentemente provienen de grados como Historia, Historia del Arte, Filosofía o Geografía. Por otro lado, el escaso reconocimiento de los recursos digitales puede estar supeditado a su desconocimiento sobre cómo integrarlos en los procesos educativos, evidenciándose así la necesidad de mejorar la formación inicial en este sentido y de integrar prácticas en las aulas universitarias que mejoren las competencias digitales de los futuros docentes.

De esta investigación se extrae una implicación educativa de gran relevancia e interés: la necesidad de mejorar el conocimiento educativo de 
los recursos tecnológicos en el aula de historia y su vinculación con un enfoque de enseñanza basado en el alumnado. Es necesario planificar intervenciones en los programas de formación del profesorado que permitan reforzar una educación histórica basada en competencias mediante la implementación de metodologías activas de aprendizaje, uso de recursos digitales y enfoques innovadores. Una intervención justificada que requiere de un mayor compromiso si tenemos en cuenta los resultados de recientes investigaciones que han señalado diferencias por sexo en relación con la intencionalidad sobre el uso de recursos tecnológicos y digitales en el aula (Gómez, Chaparro et al., 2020).

Somos conscientes de que las concepciones y experiencias escolares previas tienen un notable peso en los enfoques de enseñanza que desarrollará en el aula (González y Fuentes, 2011). Como señala Estepa (2017), los principales referentes que guían los procesos de enseñanza de los y las docentes son sus propios recuerdos que conservan de su época de estudiantes. Por eso es tan importante conocer con qué concepciones epistemológicas y metodológicas viene nuestro alumnado, para actuar sobre ellas y transformarlas. Es necesario fortalecer la formación y la aplicación de la tecnología existente para el aprendizaje y la enseñanza de la historia. Por este motivo, con este trabajo podemos partir hacia un diseño de políticas educativas a nivel nacional y, de esta manera, mejorar la calidad formativa.

La limitación principal de este estudio recae en la necesidad de un análisis cualitativo complementario mediante entrevistas y grupos de discusión con el fin de profundizar y matizar la parte cuantitativa llevada a cabo. Como prospectiva de investigación, se ve relevante estudiar el impacto de las variables planteadas en este estudio en el ámbito de la formación secundaria, y reforzar los programas de formación del profesorado en competencias digitales docentes de forma que se pueda llegar más allá del simple conocimiento de las herramientas TIC.

\section{NOTAS}

1 Esta investigación ha sido posible gracias al proyecto de I+D de «Generación de conocimiento" PGC2018-094491-B-C33 financiado por el Ministerio de Ciencia e Innovación y cofinanciado con fondos FEDER de la Unión Europea, gracias al proyecto 20638/JLI/18 financiado por la Fundación Séneca, gracias al proyecto "HistoryLab for European Civic Engagement: open e-Toolkit to train History Teachers on Digital Teaching and Learning", subvencionado por el SEPIE en la convocatoria ERASMUS + KA226 [2020-1-ES01-KA226-HE-095430], y gracias al proyecto de investigación PID2020113453RB-I00, subvencionado por la Agencia Estatal de Investigación de España (AEI/10.13039/501100011033). 


\section{REFERENCIAS BIBLIOGRÁFICAS}

Arancibia, M. M., \& Badia, A. (2015). Concepciones de profesores de secundaria sobre enseñar y aprender Historia con TIC. Revista Electrónica de Investigación Educativa, 17(2), 62-76. https://doi.org/10.22370/ ieya.2016.2.2.594

Bangdiwala, S. (1987). Using SAS software graphical procedures for the observer agreement chart. Proceedings of the SAS Users Group International Conference, 12, 1083-1088.

Beaujean, A. (2014). Latent variable modeling using $R$ : A step-by-step guide. Routledge/Taylor y Francis. https:// doi.org/10.4324/9781315869780

Claro, M., Salinas, A., Cabello-Hutt, T., San-Martín, E., Preiss, D. D., Valenzuela, S., \& Jara, I. (2018). Teaching in a Digital Environment (TIDE): Defining and measuring teachers' capacity to develop students' digital information and communication skills. Computers \& Education, 121, 162-174. https://doi. org/10.1016/j.compedu.2018.03.001

De Groot-Reuvekamp, M., Ros, A., \& Van Boxtel, C. (2018). Improving elementary school students' understanding of historical time: Effects of teaching with "Timewise". Theory and Research in Social Education, 46(1), 35-67. https://doi.or $\mathrm{g} / 10.1080 / 00933104.2017 .1357058$

Dejene, W., Bishaw, A., \& Dagnew, A. (2018). Preservice teachers' approaches to learning and their teaching approach preferences: Secondary teacher education program in focus. Cogent Education, 5(1), 1-15. https://doi.org/10.1080/23311 86X.2018.1502396
Estepa, J. (2017). Otra didáctica de la Historia para otra escuela. Universidad de Huelva.

García-Martín, J., \& García-Sánchez, J. N. (2017). Pre-service teachers' perceptions of the competence dimensions of digital literacy and of psychological and educational measures. Computers \& Education, 107, 54-67. https://doi.org/10.1016/j. compedu.2016.12.010

Gargallo, B., Garfella, P. R., Sahuquillo, P. M., Verde, I., \& Jiménez, M.Á. (2015). Métodos centrados en el aprendizaje, estrategias y enfoques de aprendizaje en estudiantes universitarios. Revista de Educación, 370, 229254. https://doi.org/10.4438/1988592X-RE-2015-370-304

George, D., \& Mallery, P. (2011). IBM SPSS Statistics 19 Step by Step: A Simple Guide and Reference. Pearson.

Gómez, C. J., Chaparro, Á., Felices, M. M., \& Cózar, R. (2020). Estrategias metodológicas y uso de recursos digitales para la enseñanza de la historia. Análisis de recuerdos y opiniones del profesorado en formación inicial. Aula Abierta, 49(1), 65-74.

Gómez, C. J., Miralles, P., RodríguezMedina, J., \& Maquilón, J. (2020). Perceptions on the procedures and techniques for assessing history and defining teaching profiles. Teacher training in Spain and the United Kingdom. Educational Studies, 47(4) 1-19. https://doi.org/10.1080/03055698 .2019.1707069

Gómez, C. J., Monteagudo, J., Moreno, J. R., \& Sainz, M. (2020). Evaluation of a gamification and flipped-classroom program used in teacher training: 
Perception of learning and outcome. PLoS ONE, 15(7), 1-19. https://doi. org/10.1371/journal.pone.0236083

Gómez, C. J., Monteagudo, J., Rodríguez Medina, J., \& Miralles, P. (2019). Evaluación de un programa de intervención sobre educación histórica en la formación inicial del profesorado de educación secundaria. En S. Alonso García, J. M. Romero Rodríguez, C. Rodríguez-Jiménez, \& J. M. Sola Reche (Eds.), Investigación, Innovación docente y TIC. Nuevos horizontes educativos (pp. 1648-1661). Dykinson.

Gómez, C. J., Rodríguez, R. A., \& Mirete, A. B. (2018). Percepción de la enseñanza de la historia y concepciones epistemológicas. Una investigación con futuros maestros. Revista Complutense de Educación, 29(1), 237-250. http://dx.doi. org/10.5209/RCED.52233

González, M., \& Fuentes, E. J. (2011). El Practicum en el aprendizaje de la profesión docente. Revista de Educación, 354, 47-70.

Guillermo, M., Cárdenas, F. A., y Hernández-Pina, F. (2018). Enfoques de enseñanza y enfoques de aprendizaje perspectivas teóricas promisorias para el desarrollo de investigaciones en educación en ciencias, Ciencia \& Educação, 24(4), 993-1012.

Hair, J. F., Black, W. C., Babin, B. J., \& Anderson, R. E. (2010). Multivariate data analysis, 7th. ed. Prentice Hall.

Hatlevik, I. K. R., \& Hatlevik, O.E. (2018). Students' evaluation of digital information: The role teachers play and factors that influence variability Human Behavior, 83, 56-63. https:// doi.org/10.1016/j.chb.2018.01.022
Kenny, D. A. (2012). Structural equation modeling. Sage.

King, F., Logan, A., \& Lohan, A. (2019). Self-study enabling understanding of the scholarship of teaching and learning: An exploration of collaboration among teacher educators for special and inclusive education. Studying Teacher Education, 15(2), 118-138. https://doi.org/10.1080/17425 964.2019.1587607

König, J., Ligtvoeta, R., Klemenza, S., \& Rothlandb, M. (2017). Effects of opportunities to learn in teacher preparation on future teachers' general pedagogical knowledge: Analyzing program characteristics and outcomes. Studies in Educational Evaluation, 53, 122-133. https://doi. org/10.1016/j.stueduc.2017.03.001

Martínez, N., Miralles, P., \& Navarro, E. (2009). Análisis de cómo los docentes plantean el inicio de las clases, desde la perspectiva del alumnado. Revista de Educación, 348, 413-433. https:// n9.cl/temoz

Miralles, P., Gómez, C. J., Arias, V., \& Fontal, O. (2019). Digital resources and didactic methodology in the initial training of History teachers. Comunicar. Media Education Research Journal, 27(2), 41-51. https://doi. org/10.3916/C61-2019-04

Miralles, P., Gómez, C. J., \& Monteagudo, J. (2019). Perceptions on the use of ICT resources and mass-media for the teaching of History. A comparative study among future teachers of SpainEngland. Educación XX1, 22(2), 187-211. https://doi.org/10.5944/ educXX1.21377

Monroy, F., Hernández-Pina, F., \& González-Geraldo, J. L. (2015). A psychometric analysis of the approaches to teaching inventory (ATI) 
and a proposal for a Spanish version (S-ATI-20). Anales de Psicología, 31(1), 172-183. https://doi.org/10.6018/ analesps.31.1.190261

Moreno-Guerrero, A. J., Aznar-Díaz, I., Cáceres-Reche, P., \& Alonso-García, S. (2020). E-learning in the teaching of mathematics: an educational experience in adult high school. Mathematics, 8(5), 840. https://doi. org/10.3390/math 8050840

Prosser, M., \& Trigwell, K. (2006). Confirmatory factor analysis of the approaches to teaching inventory. British Journal of Educational Psychology, 76(2), 405-419. https://doi. org/10.1348/000709905X43571

Ramírez-García, A., \& GonzálezFernández, N. (2016). Competencia mediática del profesorado y del alumnado de educación obligatoria en España [Media competence of teachers and students of compulsory education in Spain]. Comunicar, 24(49), 49-58.

Rivero, M. P., \& Mur, L. (2015). Aprender ciencias sociales en la web 2.0. Iber: Didáctica de las Ciencias Sociales, Geografía e Historia, 80, 30-37.

Rodríguez-Medina, J., Gómez-Carrasco, C. J., Miralles-Martínez, P., \& AznarDíaz, I. (2020). An evaluation of an intervention programme in teacher training for geography and history: A reliability and validity analysis. Sustainability, 12(8), 3124. https://doi. org/10.3390/su12083124

Rosseel, Y. (2012). Lavaan: An R package for structural equation modeling. Journal of Statistical Software, 48(2), 1-36. https://doi.org/10.18637/jss. v048.i02

Soler, M. G., Cárdenas, F. A., \& Hernández-Pina, F. (2018). Enfoques de enseñanza y enfoques de aprendizaje: perspectivas teóricas promisorias para el desarrollo de investigaciones en educación en ciencias. Ciência \& Educação (Bauru), 24(4), 993-1012.

Steiger, J. H. (1990). Structural model evaluation and modification. Multivariate Behavioral Research, 25(2), 214-12. https://doi.org/10.1207/ s15327906mbr2502_4

Stes, A., De Maeyer, S., \& Van Petegem, P. (2010). Approaches to teaching in higher education: Validation of a Dutch version of the Approaches to Teaching Inventory. Learning Environment Research, 13, 59-73. https://doi.org/10.1007/s10984-0099066-7

Thompson, M. (2019). Early childhood pedagogy in a socio-cultural medley in Ghana: Case studies in kindergarten. International Journal of Early Childhood, 51(2), 177-192. https://doi. org/10.1007/s13158-019-00242-7

Timmerman, M. E., \& Lorenzo-Seva, U. (2011). Dimensionality assessment of ordered polytomous items with parallel analysis. Psychological Methods, 16(2), 209-220. https://doi. org/10.1037/a0023353

Trigwell, K., Prosser, M., \& Ginns, P. (2005). Phenomenographic pedagogy and a revised Approaches to teaching inventory. Higher Education Research \& Development, 24(4), 349-360. https:// doi.org/10.1080/07294360500284730

Trigwell, K., \& Prosser, M. (1996). Congruence between intention and strategy in science teachers' approach to teaching. Higher education, 32(1), 77-87. https://doi.org/10.1007/ BF00139219

Ventura-León, J. L., \& CaychoRodríguez, T. (2017). El coeficiente Omega: un método alternativo para la 
estimación de la confiabilidad. Revista Latinoamericana de Ciencias Sociales, Niñez y Juventud, 15(1), 625-627. https://n9.cl/ploh1

Yunga-Godoy, D., Loaiza, M. I., RamónJaramillo, L. N., \& Puertas, L. (2016). Enfoques de la enseñanza en educación universitaria: Una exploración desde la perspectiva latinoamericana.
Profesorado. Revista de Currículum y Formación de Profesorado, 20(3), 313333. https://n9.cl/38lp3

Zurita, F., Viciana, V., Padial, R., \& Cepero, M. (2017). Levels of satisfaction to the degree of Master of senior students. Profesorado. Revista de Curriculum y Formacion de Profesorado, 21(1), 349-367. 


\section{MATERIAL SUPLEMENTARIO}

\section{Tabla S1}

Comparación del Ajuste de los Modelos Estimados Bloque II.3

\begin{tabular}{ccccccc}
\hline & $\chi^{\mathbf{2}}$ & gl & $P$ & RMSEA & CFI & TLI \\
\hline 1 Factor & 481.476 & 104 & $<.01$ & .076 & .91 & .897 \\
\hline 2 Factores & 42.542 & 103 & $<.01$ & .07 & .925 & .912 \\
\hline 3 Factores & 401.294 & 101 & $<.01$ & .069 & .929 & .915 \\
\hline 4 Factores & 282.83 & 99 & $<.01$ & .054 & .956 & .947 \\
\hline
\end{tabular}

\section{Figura S1}

Representación gráfica del modelo estructural del Bloque II.3

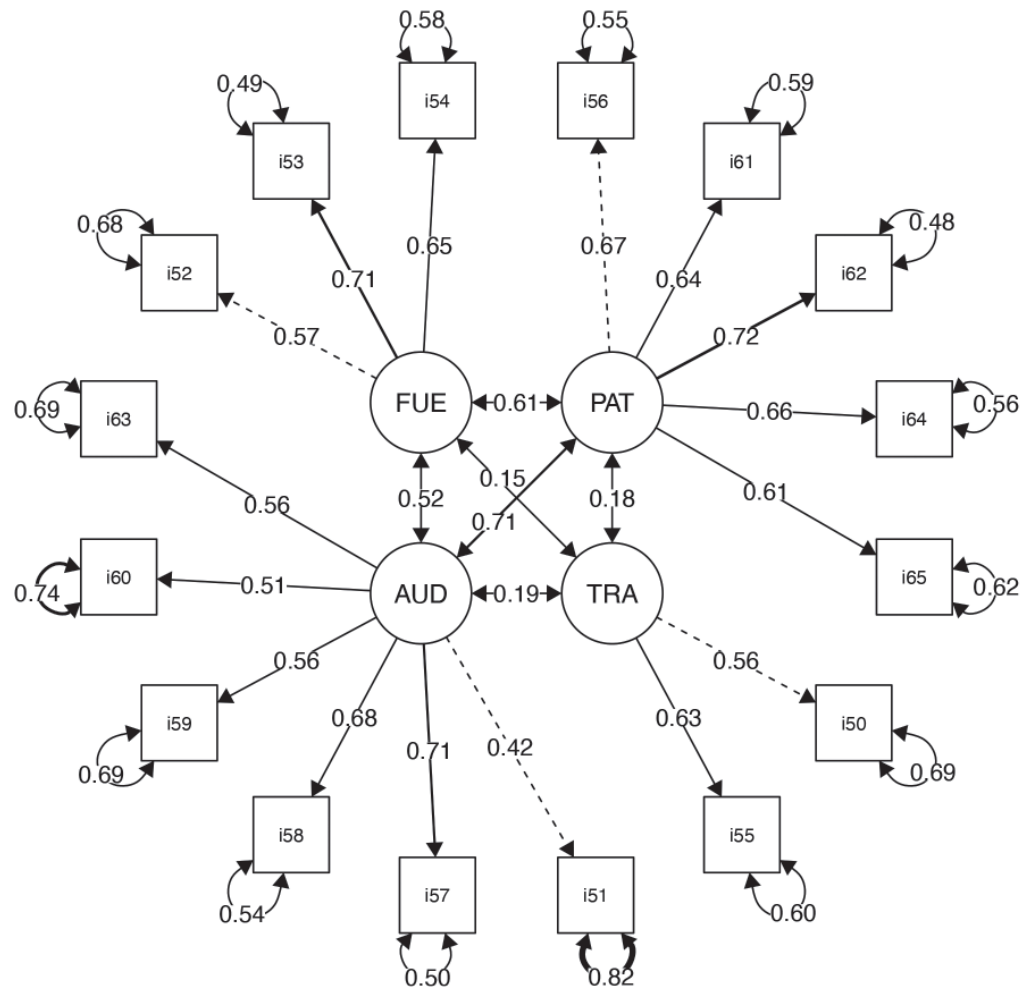




\section{PERFIL ACADÉMICO Y PROFESIONAL DE LOS AUTORES}

Cosme Jesús Gómez Carrasco. ORCID: https://orcid.org/0000-0002-92725177

Profesor Titular de Didáctica de las Ciencias Sociales de la Universidad de Murcia. Doctor por la Universidad de Castilla-La Mancha, ha sido becario postdoctoral en la la École des Hautes Études en Sciences Sociales (París), en la Universidad Carlos III de Madrid, Profesor Invitado en el Centre de Recherches Historiques, y Honorary Researcher Associate en la University College of London - Institute of Education. Sus líneas de investigación son la formación del profesorado de Ciencias Sociales, pensamiento histórico, métodos activos de aprendizaje y tecnología educativa. E-mail: cjgomez@um.es

Jairo Rodríguez-Medina. ORCID: https://orcid.org/0000-0002-6466-5525

Profesor Ayudante Doctor en el Departamento de Pedagogía de la Universidad de Valladolid. Sus líneas de investigación se centran en la evaluación de programas para la inclusión escolar, social y laboral de las personas con discapacidad intelectual y otros trastornos del neurodesarrollo, la aplicación de la metodología observacional, los métodos mixtos de investigación y el análisis de redes. E-mail: jairo. rodriguez.medina@uva.es

Álvaro Chaparro Sainz. ORCID: https://orcid.org/0000-0002-4118-9394

Profesor Ayudante Doctor de Didáctica de las Ciencias Sociales de la Universidad de Almería. Doctor por la Universidad del País Vasco, ha sido becario postdoctoral de la Université Lyon II Lumières y de la Casa de Velázquez. Sus líneas de investigación son la formación del profesorado de Ciencias Sociales, pensamiento histórico, educación patrimonial, métodos activos de aprendizaje y tecnología educativa. E-mail: alvarocs@ual.es

Santiago Alonso García. ORCID: https://orcid.org/0000-0002-9525-709X

Profesor Contratado Doctor de Didáctica y Organización Escolar de la Universidad de Granada. Doctor por la Universidad de Granada, ha desarrollado su labor académica en cinco universidades más, tres españolas (Universidad de Castilla la Mancha, Universidad de Zaragoza, Universidad de Sevilla) y dos ecuatorianas (Universidad Nacional de Chimborazo y Universidad Nacional de Educación). Sus 
líneas de investigación son la formación del profesorado en tecnología aplicada, inclusión, y orientación. E-mail: salonsog@ugr.es

Fecha Recepción del Artículo: 30. Marzo. 2021

Fecha Modificación del Artículo: 31. Mayo. 2021

Fecha Aceptación del Artículo: 03. Julio. 2021

Fecha Revisión para Publicación: 27. Septiembre. 2021 